\title{
When effective post-exposure prophylaxis of HIV infection fails - data from clinical practice
}

\author{
Justyna D. Kowalska ${ }^{1}$, Ewa Pietraszkiewicz², Ewa Firlag-Burkacka², Andrzej Horban ${ }^{1}$ \\ ${ }^{1}$ Department of Adults' Infectious Diseases, Medical University of Warsaw, Warsaw, Poland \\ ${ }^{2}$ Hospital for Infectious Diseases, HIV Outpatient Clinic, Warsaw, Poland
}

\begin{abstract}
Human immunodeficiency virus (HIV) prophylaxis with antiretroviral treatment after sexual exposure (sPEP) is an effective and safe approach; however, its influence on future exposures and, consequently, future HIV status remains under-investigated. We have evaluated the medical records of persons who received antiretroviral drugs (ARVs) as sPEP in the years 2009-2013. Cox proportional hazard models were used to identify predictors of having sexual exposure after finalising sPEP with HIV-negative status. In total 98 persons received sPEP: 37 (38\%) after unprotected men who have sex with men (MSM) intercourse, 38 (39\%) after sexual assault, and 23 (23\%) after unprotected vaginal intercourse. In 40 (41\%) cases the partner was HIV positive. Twelve persons (12\%) repeated the same pattern of exposure; median time to next exposure was 1.55 (IQR 0.78-2.43) months. In multivariate Cox models older age was increasing, and heterosexual orientation decreasing the risk of having another exposure (HR $=1.06[95 \%$ CI: $1.00-1.12 ; \mathrm{p}=0.033$ ] and $\mathrm{HR}=0.14$ [95\% CI: 0.02-1.06; $\mathrm{p}=0.057$ ], respectively). There were no HIV infections after completing sPEP, but three (3\%) persons had occasional sexual contact afterwards, resulting in HIV infection. Median time from last negative exposure to HIV infection was 1.85 (IQR 1.79-2.43) months.

In a considerable proportion of persons sPEP had no effect on behavioural patterns, mostly in those having occasional sexual contact. The risk of having another sexual exposure was higher with age and for MSM patients. For this group of persons pre-exposure prophylaxis may be a more viable method of HIV infection prophylaxis.
\end{abstract}

HIV AIDS Rev 2017; 16: 54-57

DOI: https://doi.org/10.5114/hivar.2017.65199

Key words: HIV, transmission, prophylaxis, PEP, PreP.

\section{Introduction}

Post-exposure prophylaxis (PEP) is a well-recognised and routinely used approach in both occupational and nonoccupational exposures to human immunodeficiency virus (HIV) infection.

There is a large body of evidence showing high infectiousness for various modes of sexual transmission and providing some evidence for the utilisation of antiretroviral treatment after sexual exposure (sPEP). Despite the fact that sPEP is still an underutilised prevention strategy. There is a strong need for educating and increasing awareness about such methods of HIV prevention, especially among persons or populations with high-risk behaviours.

If properly addressed sPEP should theoretically improve knowledge and interest in other prevention methods available for persons at increased risk of HIV acquisition. In a recent study three quarters of sPEP users expressed interest in pre-exposure prophylaxis with antiretrovirals (PreP). How-

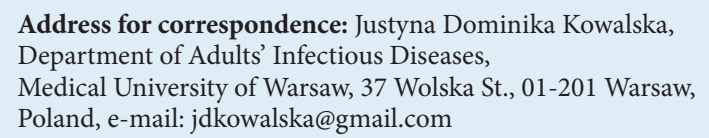

Address for correspondence: Justyna Dominika Kowalska, Department of Adults' Infectious Diseases,

Medical University of Warsaw, 37 Wolska St., 01-201 Warsaw,

Poland, e-mail: jdkowalska@gmail.com

\section{Article history:}

Received: 03.10.2016

Received in revised form: 30.10 .2016

Accepted: 01.11.2016

Available online: 18.01 .2017
International Journal of HIV-Related Problems

HIV \& AIDS

R e vi e w 
Table 1. Comparison of baseline characteristics for patients having and not having repeated exposure after post sexual-exposure prophylaxis (SPEP) care

\begin{tabular}{|c|c|c|c|}
\hline Characteristic & $\begin{array}{l}\text { Repeated exposure, } \\
\qquad n=12\end{array}$ & $\begin{array}{l}\text { No repeated exposure, } \\
\qquad n=86\end{array}$ & $p$ value \\
\hline Gender (male), $n$ (\%) & $9(75.0)$ & $43(50.0)$ & 0.10 \\
\hline Age in years, median (IQR) & $33.9(28.6-39.3)$ & $28.0(23.2-35.4)$ & 0.27 \\
\hline Sexual orientation MSM, $n$ (\%) & $7(58.3)$ & $30(34.9)$ & 0.12 \\
\hline Source HIV positive, $n$ (\%) & $3(27.3)$ & $28(32.6)$ & 0.72 \\
\hline NDL, $n(\%)$ & $3(25.0)$ & $35(40.7)$ & 0.29 \\
\hline \multicolumn{4}{|l|}{ Type of exposure, $n(\%)$} \\
\hline MSM anal sex & $7(58.3)$ & $25(29.1)$ & \multirow{4}{*}{0.06} \\
\hline MSM oral sex & $0(0.0)$ & $5(5.8)$ & \\
\hline Vaginal sex & $4(33.3)$ & $19(22.1)$ & \\
\hline Sexual assault & $1(8.3)$ & $37(43.0)$ & \\
\hline
\end{tabular}

MSM - men who have sex with men

ever, the use of both interventions in different risk groups and in clinical settings needs to be further discussed. For example their utilisation in serodiscordant couples, where an HIV-positive partner is on effective antiretroviral treatment, needs to be re-evaluated.

The use of antiretroviral treatment in HIV uninfected individuals is generally considered as a safe method, both for older and newer agents. Although most observed adverse drug reactions are mild and of reversible nature, their occurrence is much higher in HIV-negative than HIV-positive persons. This can lead to poorer adherence to treatment and decrease the net benefit from such prevention methods.

Another concern is the effect of sPEP on sexual risk perception and future sexual behaviours. Studies investigating this vital problem showed inconsistent results, which reflects the difference in both design and target population. Studies report SPEP users to be more likely to present high-risk behaviours, but whether sPEP has a preventive influence towards such behaviours remains uncertain.

The influence of sPEP on future exposures and, in consequence, future HIV status, especially in the clinical setting, remains under-investigated. Therefore, we have evaluated medical records of persons who received sPEP in an HIV Outpatient Clinic in Warsaw in the past five years.

\section{Material and methods}

Medical records of persons consulted at the HIV Outpatient Clinic of the Hospital for Infectious Diseases in Warsaw after unprotected sexual intercourse were reviewed. Only patients who received antiretroviral therapy as HIV prophylaxis for sexual exposure were included into the study. Five consecutive years (2009-2013) were reviewed.

The routine consultation after exposure to HIV infection including counselling and individual risk assessment was performed by an infectious disease specialist working in the clinic. It was followed by a decision on prescribing and the choice of antiretroviral drugs. Testing for HIV infection was performed at baseline, six weeks, and three months. At each visit the patient was advised on safe sex methods and given an opportunity to discuss any doubts on the risk of acquiring HIV.

For the study, indications for starting nPEP were grouped as following: men who have sex with men (MSM) oral intercourse, MSM anal intercourse, heterosexual vaginal intercourse, and sexual assault (irrespective of sexual orientation). Information on adverse drug reactions was evaluated as part of another project but was available for this analysis .

In statistical analyses $\chi^{2}$ and Kruskal-Wallis tests were used for group comparisons. Cox proportional hazard models were used to identify predictors of having sexual exposure after finalising sPEP. Variables tested in univariate analyses were age, gender, sexual orientation, HIV status of sexual partner, and adverse reaction to any medication used in sPEP. A multivariable model included all listed variables. A confidence interval (CI) of $95 \%$ was accepted. All analyses were performer using SAS version 9.3 (SAS Institute, Cary, NC).

\section{Results}

In total 98 persons received sPEP, 37 (38\%) MSM after unprotected intercourse, 38 (39\%) MSM after sexual assault, and $23(23 \%)$ heterosexual persons after unprotected vaginal intercourse. In 40 (41\%) cases the sexual partner was known to be HIV positive.

Twelve persons (12\%) repeated the same pattern of sexual exposure, five through vaginal and seven through MSM anal intercourse. Eight exposures were with an occasional partner (two with an HIV-positive partner), four in serodiscordant couples. Median time from the first to next sex- 
Table 2. Cox proportional hazard models for the risk of having next sexual exposure

\begin{tabular}{|c|c|c|c|c|c|c|}
\hline \multirow{2}{*}{ Factor } & \multicolumn{3}{|c|}{ Univariate } & \multicolumn{3}{|c|}{ Multivariate } \\
\hline & Hazard ratio & $95 \% \mathrm{Cl}$ & $p$ value & Hazard ratio & $95 \% \mathrm{Cl}$ & $p$ value \\
\hline \multicolumn{7}{|l|}{ Gender } \\
\hline Female & 1.00 & - & - & 1.00 & - & - \\
\hline Male & 2.18 & $0.59-8.14$ & 0.244 & 0.74 & $0.11-4.88$ & 0.755 \\
\hline \multicolumn{7}{|l|}{ Age } \\
\hline Per 1 year older & 1.04 & $0.99-1.09$ & 0.116 & 1.06 & $1.00-1.12$ & 0.033 \\
\hline Per 10 years older & 1.46 & $0.91-2.35$ & 0.116 & 1.84 & $1.05-3.22$ & 0.033 \\
\hline \multicolumn{7}{|c|}{ Adverse reaction to any medication used in SPEP } \\
\hline No & 1.00 & - & - & 1.00 & - & - \\
\hline Yes & 0.63 & $0.17-2.33$ & 0.484 & 0.50 & $0.12-2.00$ & 0.327 \\
\hline \multicolumn{7}{|l|}{ Sexual orientation } \\
\hline MSM & 1.00 & - & - & 1.00 & - & - \\
\hline Heterosexual & 0.40 & $0.12-1.26$ & 0.118 & 0.14 & $0.02-1.06$ & 0.057 \\
\hline \multicolumn{7}{|c|}{ Sexual partner HIV status } \\
\hline Unknown & 1.00 & - & - & 1.00 & - & - \\
\hline HIV (+) & 0.838 & $0.22-3.17$ & 0.794 & 0.33 & $0.07-1.61$ & 0.170 \\
\hline
\end{tabular}

SPEP - post sexual-exposure prophylaxis, HIV - human immunodeficiency virus, MSM - men who have sex with men

ual exposure consulted in the clinic was 1.55 (interquartile range, IQR: 0.78-2.43) months. Six persons (6\%) received sPEP again.

In general, persons reporting repeated exposure were more likely to be older, male, and MSM. Persons having MSM anal sex and vaginal sex were more likely to repeat the exposure. However, none of these differences reached statistical significance (Table 1).

In the multivariate Cox model older age was increasing, and heterosexual orientation decreasing the risk of having another sexual exposure $(\mathrm{HR}=1.06$ [95\% CI: 1.00-1.12; $p=0.033$ ] and HR $=0.14$ [95\% CI: 0.02-1.06; $p=0.057$, respectively) (Table 2 ).

There were no HIV infections after completing sPEP, but three (3\%) persons had occasional sexual contact afterwards resulting in HIV infection. Median time from last negative exposure until HIV infection was 1.85 (IQR 1.79-2.43) months.

\section{Discussion}

In one out of ten persons sPEP had no effect on behavioural patterns, mostly in those having occasional contact. The risk of having another sexual exposure was higher with age and for MSM patients. For this group of persons pre-exposure prophylaxis may be a more viable method of HIV infection prophylaxis.

Numerous studies have reported an increase in the number of sexual exposures to HIV in recent years, mostly through high-risk behaviours and unprotected sexual intercourse, especially among MSM. A recent molecular phylogenetic analysis by Drescher et al. showed that treatment naïve
HIV-positive MSM are the main group transmitting HIV in the British Columbian population . In fact, MSM sexual contact is the main mode of transmission in high-income countries. In Poland MSM remains an increasing and recently dominating group of newly diagnosed HIV persons. Also, in our work MSM were associated with higher risk of repeated sexual exposure, and the only HIV infections that were reported in our study occurred as a result of MSM sexual intercourse.

As of today many different approaches have been proposed in response to the observed situation. These include better testing strategies, increased linkage to care, offering antiretroviral treatment to HIV-positive patients for transmission risk reduction, and using antiretrovirals in the HIV-negative population as SPEP or PreP. Although effective in clinical studies, none of these methods was proven to work in clinical settings as a single intervention. Therefore, combination prevention strategy that includes all elements, as well as STI treatment and structural behavioural interventions, seems to be the only feasible approach. Moreover, any planned intervention needs to be re-evaluated in real-life settings. There are far more factors associated with sexual behaviours: pattern of illicit drug or legal highs use, program availability, cultural diversity, and many others. In this light, any experience in addressing the risk of HIV exposure in clinical practice is a vital addition to strategy planning.

There are some limitations to our work, which need to be mentioned. First of all, the retrospective nature of this work should be considered while interpreting the results. The number of HIV infections could be underestimated, but all persons diagnosed with HIV in the central region of Po- 
land are referred and registered in the Warsaw Outpatient Clinic. Their earlier records from sPEP counselling are included in both electronic and paper documentation. Finally, because we had no access to the information on HIV-positive partner treatment, we were not able to describe the protective effect of sPEP separately from these vital factors.

An important limitation for sPEP is the fact that it can only be prescribed in clinical centres with specialists experienced in HIV treatment. On the other hand, in most European countries it is available free of charge, unlike PreP.

Post-exposure prophylaxis is an emergency medical assistance and as such will always have its place in any prevention program.

\section{Conflict of interest}

The author's declared no potential conflicts of interest with respect to the research, authorship, and/or publication of this article.

\section{References}

1. Roland ME. Enhancing the potential benefits of HIV post-exposure prophylaxis. AIDS 2006; 20: 1889-1890.

2. Lunding S, Katzenstein TL, Kronborg G, et al. The Danish PEP registry: experience with the use of postexposure prophylaxis (PEP) following sexual exposure to HIV from 1998 to 2006. Sex Transm Dis 2010; 37: 49-52.

3. Casalino E, Choquet C, Leleu A, et al. Trends in condom use and risk behaviours after sexual exposure to HIV: a seven-year observational study. PLoS One 2014; 9: e104350.

4. Halperin DT. Heterosexual anal intercourse: prevalence, cultural factors, and HIV infection and other health risks, Part I. AIDS Patient Care STDS 1999; 13: 717-730.

5. Baggaley RF, White RG, Boily MC. HIV transmission risk through anal intercourse: systematic review, meta-analysis and implications for HIV prevention. Int J Epidemiol 2010; 39: 1048-1063.

6. Roland ME. Postexposure prophylaxis after sexual exposure to HIV. Curr Opin Infect Dis 2007; 20: 39-46.

7. McDougal SJ, Alexander J, Dhanireddy S, et al. Non-occupational post-exposure prophylaxis for HIV: 10-year retrospective analysis in Seattle, Washington. PLoS One 2014; 9: e105030.

8. Sonder GJ, van den Hoek A, Regez RM, et al. Trends in HIV postexposure prophylaxis prescription and compliance after sexual exposure in Amsterdam, 2000-2004. Sex Transm Dis 2007; 34: 288-293.

9. Minas B, Laing S, Jordan H, et al. Improved awareness and appropriate use of non-occupational post-exposure prophylaxis (nPEP) for HIV prevention following a multi-modal communication strategy. BMC Public Health 2012; 12: 906.

10. Jain S, Gregor C, Krakower D, et al. Attitudes and interest toward HIV pre-exposure prophylaxis among participants using HIV nonoccupational post-exposure prophylaxis. ID Week; October 8-12, 2014; Philadelphia. Abstract Nr 1523.

11. Loutfy MR, Wu W, Letchumanan M, et al. Systematic review of HIV transmission between heterosexual serodiscordant couples where the HIV-positive partner is fully suppressed on antiretroviral therapy. PLoS One 2013; 8: e55747.

12. Vigil KJ, Simons P, Luna K, et al. Raltegravir plus tenofovir DF and emtricitabine for non-occupational postexposure prophylaxis (nPEP): African-Americans are at higher risk of non-completion of nPEP. ID Week; October 8-12, 2014; Philadelphia. Abstract Nr 1529.
13. Mandala J, Nanda K, Wang M, et al. Liver and renal safety of tenofovir disoproxil fumarate in combination with emtricitabine among African women in a pre-exposure prophylaxis trial. BMC Pharmacology \& Toxicology 2014; 15: 77.

14. Tosini W, Muller P, Prazuck T, et al. Tolerability of HIV postexposure prophylaxis with tenofovir/emtricitabine and lopinavir/ritonavir tablet formulation. AIDS 2010; 24: 2375-2380.

15. Pietraszkiewicz E, Firlag-Burkacka E, Horban A, et al. The suspected unexpected and serious adverse events of antiretroviral drugs used as HIV prophylaxis in HIV uninfected persons. J Int AIDS Soc 2014; 17 (4 Suppl 3): 19733.

16. Bryant J, Baxter L, Hird S. Non-occupational postexposure prophylaxis for HIV: a systematic review. Health Technol Assess 2009; 13: iii, ix-x, 1-60.

17. Ford N, Irvine C, Shubber Z, et al. Adherence to HIV postexposure prophylaxis: a systematic review and meta-analysis. AIDS 2014; 28 : 2721-2727.

18. Donnell D, Mimiaga MJ, Mayer K, et al. Use of non-occupational post-exposure prophylaxis does not lead to an increase in high risk sex behaviors in men who have sex with men participating in the EXPLORE trial. AIDS Behav 2010; 14: 1182-1189.

19. Schechter M, do Lago RF, Mendelsohn AB, et al. Behavioral impact, acceptability, and HIV incidence among homosexual men with access to postexposure chemoprophylaxis for HIV. J Acquir Immune Defic Syndr 2004; 35: 519-525.

20. Reback CJ, Fletcher JB, Shoptaw S, et al. Methamphetamine and other substance use trends among street-recruited men who have sex with men, from 2008 to 2011. Drug Alcohol Depend 2013; 133: 262-265.

21. Poon AF, Joy JB, Woods CK, et al. The impact of clinical, demographic and risk factors on rates of HIV transmission. A population-based phylogenetic analysis in British Columbia, Canada. J Infect Dis 2015; 211: 926-935.

22. Drescher SM, von Wyl V, Yang WL, et al. Treatment-naive individuals are the major source of transmitted HIV-1 drug resistance in men who have sex with men in the Swiss HIV Cohort Study. Clin Infect Dis 2014; 58: 285-294.

23. Rosinska M, Janiec J, Niedzwiedzka-Stadnik M. Increase of new HIV diagnoses among men who have sex with men in Poland, 2000 to 2011. Euro Surveill 2013; 18: 20642.

24. Rosinska M, Marzec-Bogustawska A, Janiec J, et al. High percentage of recent HIV infection among HIV-positive individuals newly diagnosed at voluntary counseling and testing sites in Poland. AIDS Res Hum Retroviruses 2013; 29: 805-813.

25. Muessig KE, Smith MK, Powers KA, et al. Does ART prevent HIV transmission among MSM? AIDS 2012; 26: 2267-2273.

26. Ankiersztejn-Bartczak M, Firlag-Burkacka E, Czeszko-Paprocka H, et al. Factors responsible for incomplete linkage to care after HIV diagnosis: preliminary results from the Test and Keep in Care (TAK) project. HIV Med 2015; 16: 88-94.

27. Fernandez-Balbuena S, Belza MJ, Castilla J, et al. Awareness and use of nonoccupational HIV post-exposure prophylaxis among people receiving rapid HIV testing in Spain. HIV Med 2013; 14: 252-257. 